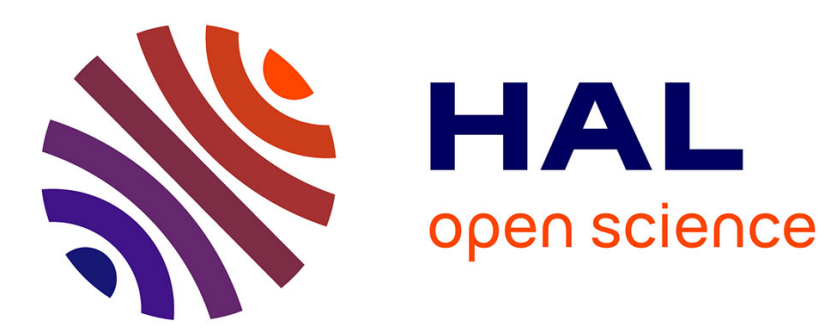

\title{
Wall-Normal Velocity Correlations in a Zero Pressure Gradient Turbulent Boundary Layer
}

Sylvain Morilhat, François Chedevergne, Francis Micheli, Frank Simon

\section{To cite this version:}

Sylvain Morilhat, François Chedevergne, Francis Micheli, Frank Simon. Wall-Normal Velocity Correlations in a Zero Pressure Gradient Turbulent Boundary Layer. Journal of Fluids Engineering, 2020, 142 (8), pp.081302. 10.1115/1.4046873 . hal-02969156

\section{HAL Id: hal-02969156 https://hal.science/hal-02969156}

Submitted on 16 Oct 2020

HAL is a multi-disciplinary open access archive for the deposit and dissemination of scientific research documents, whether they are published or not. The documents may come from teaching and research institutions in France or abroad, or from public or private research centers.
L'archive ouverte pluridisciplinaire HAL, est destinée au dépôt et à la diffusion de documents scientifiques de niveau recherche, publiés ou non, émanant des établissements d'enseignement et de recherche français ou étrangers, des laboratoires publics ou privés. 


\title{
Wall-normal velocity correlations in a zero pressure gradient turbulent boundary layer
}

\author{
Sylvain Morilhat \\ Research Scientist \\ ONERA \\ François Chedevergne* \\ Research Scientist \\ ONERA \\ Department Multi-Physics for Energetics \\ 2 avenue Edouard Belin \\ 31055, Toulouse Cedex 4 \\ Email: francois.chedevergne@onera.fr
}

Francis Micheli
Research Scientist
ONERA

Frank Simon

Research Scientist

ONERA

\begin{abstract}
An experimental campaign dedicated to the characterization of the wall-normal velocity correlations in a zero pressure gradient turbulent boundary layer was performed. A double set of Laser Doppler Velocimetry benches were used to access two-point two-time correlations of the wall-normal velocity. The measurements analysis confirms several important hypotheses classically made to model wall pressure spectra from the velocity correlations.
\end{abstract}

*Address all correspondence related to ASME style format and figures to this author. 
In particular, the ratio of the wall-normal Reynolds stress to the turbulent shear stress is confirmed to exhibit a large plateau in the logarithmic region. In addition, Taylor's hypothesis of frozen turbulence is well recovered for the wall-normal velocity fluctuations. The convection velocity for the wall-normal velocity fluctuations is also shown to evolve across the boundary layer, according to the mean velocity profile. Furthermore, the decorrelation time scale of velocity correlations appears to be increasing throughout the boundary layer thickness in accordance with the increase of the convection velocity. The results obtained with this original campaign will help improving models for wall pressure spectra, especially those based on the resolution of the Poisson equation for the pressure for which the wall pressure correlations are related to the wall-normal velocity correlations.

\section{INTRODUCTION}

Turbulent boundary layers produce wall pressure fluctuations that may cause undesirable structure vibrations which in turn can generate acoustic radiations. To evaluate the stochastic response of vibrating structures to wall pressure excitation, the space/time correlation function, or equivalently the wave-number/angular-frequency spectrum, is required. Among the different methods developed to model the pressure spectrum $\Phi_{p p}(\mathbf{k}, \omega)$, where $\mathbf{k}$ is the wave-number and $\omega$ the angular frequency, recent efforts were focused on the resolution of the Poisson equation for the pressure fluctuations. They are then directly related to the velocity correlations of the turbulent flow. This approach was initiated by Kraichnan [1] and continued by Panton and Linebarger [2]. However, these authors were limited by the available computational power. More recently, Slama et al. [3], following Peltier and Hambric [4] found a solution to the Poisson equation using turbulent velocity fluctuations as source terms for wall pressure correlations in the space-time domain, the pressure spectrum being then obtained by Fourier transform. Morilhat et al. $[5,6]$ derived a model from the work of Lysak [7] and Aupoix [8] that enables to directly access the pressure spectrum from the resolution of the Poisson equation in the spectral domain. Those methods rely on several hypothesis concerning wall pressure and velocity correlations in a turbulent boundary layer. Even if DNS databases, e.g. the work by Sillero et al. [9], can provide accurate predictions and 
can serve for modeling, their high computational costs for high Reynolds number configurations remain a major obstacle. To the authors knowledge, there is no available experimental data that could be used to develop a model for $\Phi_{p p}(\mathbf{k}, \omega)$ based on the resolution of the Poisson equation. Such an experiment ideally requires joint measurements of both the spatial correlations of the velocity and of the wall pressure fluctuations. Nevertheless, by combining experimental data coming from two sets of different experiments for velocity correlations and pressure fluctuations, models such as those of Morilhat et al. [5] and Slama et al. [3] can be assessed on critical points. Many experiments provide accurate data for pressure fluctuations [10-13] but very few can be used to validate hypothesis made on velocity correlations.

Measurements of turbulent wall pressure fluctuations can only be obtained in a low-noise facility such as an anechoic wind-tunnel. Conversely, velocity fluctuations are only weakly affected by acoustical disturbances and can be obtained in a standard wind-tunnel without specific acoustic treatment. The purpose of the present work is to provide experimental data of turbulent velocity fluctuations that will be used to consolidate $\Phi_{p p}(\mathbf{k}, \omega)$ models.

To measure spatial correlations of turbulent velocity fluctuations, three distinct experimental set-ups using HWA (hot-wire anemometry), LDV (Laser Doppler Velocimetry) or PIV (Particule Image Velocimetry) techniques were used over the years. Early contributions from Favre et al. [14], Tritton [15] and Grant [16] make use of HWA measurements for the spatial correlations. Although major results were gained from these experiments, the intrusive nature of the hot-wires limits the extent of this technique. In particular, difficulties are encountered when measuring longitudinal correlations due to the wake of the upstream hot-wire. In the present context, spatial correlation measurements are realized using LDV measurements. Among the dedicated experiments inventoried in the literature and relying on the use of LDV, three kinds of set-ups may be distinguished.

The first two set-ups only use one velocimeter, rendering these two techniques cheaper but not necessarly easy to implement. Belmabrouk and Michard [17] used a 2D emitting head with four laser beams, generating two measurement volumes (MV). One is kept fixed while the second is moved by the use of rotating mirrors. This method was used to study grid turbulence and jet flows. The second technique, only necessitating a unique velocimeter, consists in the generation 
of an elongated MV using a set of long focal lens. The spacing between the fringes is not constant along the MV. With two receiving heads focalized at two different locations in the elongated MV, a spatial correlation can be deduced. This technique was employed by Neumann et al. [18] to study the wake flow behind a cylinder and Fraser et al. [19] to study grid turbulence. Although these two techniques only required a unique velocimeter, some limitations inevitably occur. In particular, in terms of accuracy, a two velocimeters set-up is in essence preferable.

A set-up involving two velocimeters was used by Bech et al. [20] to study a Couette flow, Furuichi et al. [21], Benedict and Gould [22] to study the detached flow behind a step and Trimis and Melling [23] for a jet flow configuration. In a boundary layer context, Ölçmen and Semih [24] made two-point correlations measurements of the wall-normal fluctuating velocity in the vicinity of the wall. In the present study, a similar set-up is adopted to access the spatial correlations of the wall-normal velocity component in a $3 \mathrm{D}$ field.

PIV was also widely used to study velocity correlations. The ability to perform measurements in a plane is a major advantage over LDV which gives only acess to one-point measurements. However, until recently and the development of Time-Resolved PIV and Tomographic PIV [25], the frame rate was limited to about $10 \mathrm{~Hz}$. Therefore, only spatial characterizations were achieved with PIV. At first, only Reynolds stresses measurements were performed, e.g in the work by Meinhart and Adrian [26]. Later, spatial correlation functions were studied by Kahler [27], Ganapathisubramani et al. [28] and Adrian et al. [29].

The article is organized as follows. After a brief preamble where notations are introduced, section 3 presents the experimental setup and the measurement techniques involved in the present study. Although only a small extract of representative data is illustrated in the paper, a complete description of all the measurements is made to show the extent of the study. Section 4 provides uncertainty assessments for the velocity correlations and the derived integral scales. Then in section 5, an analysis of two-point two-time velocity correlations is performed through some illustrative examples. Spatial correlations are compared to results from a DNS database [9]. Temporal damping of the measured correlations is then extracted and compared to the modeling used by Morilhat 
et al. $[5,6]$.

At last, section 6 concludes and gives some perspectives to complement the present work.

\section{PREAMBLE}

Models providing the angular frequency spectrum $\Phi_{p p}(\omega)$ through the resolution of a Poisson equation for the pressure, for instance Slama et al. [3] or Morilhat et al. [5], relate the space-time correlation of the wall pressure fluctuations $\left\langle p^{\prime}(\mathbf{x}, t) p^{\prime}(\mathbf{x}+\mathbf{r}, t+\tau)\right\rangle$ to the two-point two-time spatial correlation of the wall-normal velocity $\left\langle v^{\prime}(\mathbf{x}, t) v^{\prime}(\mathbf{x}+\mathbf{r}, t+\tau)\right\rangle$. For the zero pressure gradient boundary layers considered, the boundary layer thickness $\delta$ increases slowly in the streamwise direction and the velocity field can be reasonably assumed as homogeneous in a plane parallel to the wall. For this reason the two-point two-time spatial correlation is expected to depend only on $y, \mathbf{r}$ and $\tau$. For the sake of compactness, the following notation is introduced:

$$
S_{v v}[y](\mathbf{r}, \tau)=\left\langle v^{\prime}(\mathbf{x}, t) v^{\prime}(\mathbf{x}+\mathbf{r}, t+\tau)\right\rangle
$$

The associated wave-number frequency spectrum $\Phi_{v v}[y](\mathbf{k}, \omega)$ is deduced from $S_{v v}[y](\mathbf{r}, \tau)$ using a direct Fourier transform. In the following, the argument $y=y_{0}$ will be dropped for the sake of clarity and the values $y_{0}$ will be explicitly stated when needed.

The measurements realized in this study give access to two-point two-time correlations $S_{v v}(\mathbf{r}, \tau)$. In the following, we use notations $S_{v v}(\mathbf{r})$ or $\Phi_{v v}(\mathbf{k})$ to designate the two-point correlation with $\tau=0$ or the corresponding space Fourier transform. Similarly, the one-point correlation is $S_{v v}(\tau)=$ $S_{v v}(\mathbf{0}, \tau)$ and the angular-frequency spectrum is $\Phi_{v v}(\omega)=\Phi_{v v}(\mathbf{0}, \omega)$. 
The LMA model of Morilhat et al. [5] reads:

$$
\begin{aligned}
& \Phi_{p p}(\mathbf{k}, \omega)=\int_{0}^{\infty} \Phi_{P} \Phi_{T} \Phi_{A} \Phi_{D} d y \\
& \Phi_{P}=\rho\left(\frac{\partial U}{\partial y}\right)^{2} l_{e}^{5}\left\langle v^{\prime} v^{\prime}\right\rangle \\
& \Phi_{T}=A_{V K} \frac{k_{x}^{2}+k_{z}^{2}}{\left(1+\mathbf{k}^{2} l_{e}^{2}\right)^{17 / 6}} \\
& \Phi_{A}=2 \pi \frac{\tau_{S} / \pi}{1+\tau_{S}^{2}\left(\omega+U k_{x}\right)^{2}} \\
& \Phi_{D}=e^{-2|\mathbf{k}| y}
\end{aligned}
$$

where $A_{V K}$ is a normalization constant coming from the use of the Von Kármán spectrum.

The LMA model relies on several closure relations for the Reynolds stress $\left\langle v^{\prime} v^{\prime}\right\rangle=S_{v v}(\mathbf{0}, 0)$, the isotropic correlation length scale $l_{e}$, the Von Kármán spectrum model, the temporal damping $\tau_{S}$. All those quantities come from the modeling of the two-point two-time correlation $S_{v v}(\mathbf{r}, \tau)$.

The objective of the present experiments is to give access to those quantities in order to validate the closure relations or to propose more advanced models if needed. Such data can serve other models for wall pressure spectrum [3] that also relate $\Phi_{p p}(\omega)$ to $S_{v v}(\mathbf{r}, \omega)$.

Measurements of correlations $S_{v v}(\mathbf{r}, \tau)$ in a boundary layer shall confirm the relevance of Taylor's hypothesis for wall normal fluctuations and give access to the convection velocity $U_{c}$ and the temporal decay $\tau_{S}$. In addition, correlation length scales shall be extracted from the measurements for each direction which will characterize the anisotropic behavior of the wall-normal fluctuations.

\section{EXPERIMENTAL SETUP}

\subsection{Wind tunnel}

Experiments were conducted in a wind tunnel of Eiffel type. The $0.4 \mathrm{~m} \times 0.4 \mathrm{~m}$ test section is $1.8 \mathrm{~m}$ long while optical access only cover $1.2 \mathrm{~m}$. The test section equipped with optical access is visible on figure 1. No models were introduced in the test section of the wind tunnel. In order to get sufficiently large boundary layer thicknesses, the boundary layers that develop on the walls of the 
wind tunnel, from the convergent up to the measuring test section, were considered. In particular, all the measurements were made in the turbulent boundary layer of the top wall of the wind-tunnel, $1.1 \mathrm{~m}$ after the convergent.

\subsection{Velocity measurement apparatus}

Measurements were performed using two independent LDV systems. Two different wavelengths were used : $488 \mathrm{~nm}$ and $561 \mathrm{~nm}$. Thus the velocimeters will be referred as "blue" and "yellow". Each velocimeter is mounted on a three axis translation stage, both translation stages being completely independent, and the spatial resolution is around $25 \mu \mathrm{m}$.

The blue laser source is a Coherent Genesis MX SLM $488 \mathrm{~nm}$ solid state laser. It can provide a $2 \mathrm{~W}$ continuous beam but only $0.5 \mathrm{~W}$ were used. The emitting head is a Dantec 60X with four optical fibers allowing to measure the longitudinal and normal components of the velocity. The source and the emission heads are connected using a color separator FiberFlow Dantec 9060X0411. Only one component of the velocity can be measured. The switch between components is realized through a change of the used fibers pair.

The yellow laser source is an Oxxius $561 \mathrm{~nm}$ solid state laser emitting a $300 \mathrm{~mW}$ continuous beam. The laser is directly connected on a modified Disa SSX-V emitting head. The receiving head is a Dantec 90SSX0341. Both set-ups are used in forward scattering. Characteristics of the two velocimeters are recapped in table 1.

The optical fibers of the receiving heads are connected to two photomultipliers Photonis XP2020, these latter being connected to a signal processor TSI FSA 4000. The signal processor is driven by the Flowsizer software (v 2.0.4.0).

Atomized DEHS oil, yielding droplets around $200 \mathrm{~nm}$ in diameter, is used as a tracer. The droplets are generated with a Topas atomizer aerosol generator ATM $210 \mathrm{H}$. The atomizer is connected to a network of perforated pipes placed in front of the convergent of the wind-tunnel. This set-up ensured a good and constant seeding during the whole experimental campaign.

The relative position of the velocimeters must be calibrated daily in order to guaranty its precision. An instrument-holder carrying a calibrated hole of $50 \mu \mathrm{m}$ diameter is placed inside the 
wind tunnel, normal to the transverse direction. Both velocimeters are aligned on this perforation using the diffraction of the laser beams through the hole. Using this procedure, the relative error in the longitudinal and normal directions is about $25 \mu \mathrm{m}$ while the error in the transverse direction is about $1 \mathrm{~mm}$.

\subsection{Experimental conditions and measurement domain}

Several series of measurements were made to give access to different types of data. First, one point measurements were performed. Mean and variance profiles $\left(U(y),\left\langle u^{\prime 2}\right\rangle(y),\left\langle v^{\prime 2}\right\rangle(y)\right.$ and $\left.\left\langle u^{\prime} v^{\prime}\right\rangle(y)\right)$ were measured for eight values of the freestream velocity $U_{e} \in[25 ; 60] \mathrm{m} / \mathrm{s}$. The corresponding range of Reynolds numbers based on the momentum thickness is $\mathcal{R}_{\theta} \in[2800,10500]$. The characteristics of these boundary layers are recapped in table 2 . The friction velocity $u_{\tau}$ was determided using the Clauser chart method [30,31]. The main objective of this first set of measurements was to explore the effect of the variation of $\mathcal{R}_{\theta}$ on the ratio $-\left\langle u^{\prime} v^{\prime}\right\rangle /\left\langle v^{\prime 2}\right\rangle$. In most of the RANS computations, only the Reynolds stress $\left\langle u^{\prime} v^{\prime}\right\rangle$ is accurately described and thus in the LMA model [5], and similarly for Aupoix [8] and Lysak [7], $\left\langle v^{\prime 2}\right\rangle$ is deduced from $\left\langle u^{\prime} v^{\prime}\right\rangle$. The measurements were obtained using the two MV of the velocimeters localized at the same point and oriented such that the longitudinal and the wall-normal velocity components were measured.

Case $U_{e}=50 \mathrm{~m} / \mathrm{s}$ was investigated in more details in a second set of measurements. Twopoint two-time correlations of the turbulent velocity fluctuations were measured using the two velocimeters. Only the wall-normal component of the velocity was measured providing spatial correlations $S_{v v}(\mathbf{r}, \tau)$. One of the $\mathrm{MV}$ is fixed at position $\mathbf{x}=\mathbf{x}_{\mathbf{0}}$ with an altitude in the boundary layer given by $y_{0}$. Let $r_{x}, r_{y}$ and $r_{z}$ designate the coordinates of the distance vector between the two MV. For each altitude $y=y_{0}$, one-dimensional profiles, for which two components of vector $\left(r_{x}, r_{y}, r_{z}\right)$ are set to zero, and bi-dimensional fields, where only one component $r_{x}, r_{y}$ or $r_{z}$ is set to zero, were measured. The different exploration lengths in each direction $D_{x}, D_{y}$ and $D_{z}$ are summarized in table 3.

Obviously, since the correlation lengths decrease as $y_{0}$ tends to zero, the exploration lengths 
provided in table 3 for the $1 \mathrm{D}$ profiles correspond to the maximum lengths used at the edge of the boundary layer. These maximum lengths were estimated a priori using the turbulence lengthscales [5]. Concerning the 2D fields of spatial correlations, choices for the maximal lengths result from a compromise. Those lengths are yet large enough for two-point one-time correlations. For one-dimensional cases, 24 different values of $y_{0}$ were considered while only 6 were retained for bidimensional explorations. For each measurement, the recording time is such that full convergence is reached, as explained in section 3. The number of acquired spatial correlations is given in table 4.

\section{UNCERTAINTIES ASSESSEMENT}

To assess the uncertainty of the LDV measurements, a specific procedure was derived. Two aspects were checked: convergence and repeatability of the measurements. Attention is mainly focused on altitude $y / \delta=0.5$ since it corresponds to the location of the maximal contribution to the convective part of the wall pressure spectrum according to the LMA model [5]. In comparison, the wall and wake regions contributions are far less significant.

\subsection{Convergence}

As reminded hereinabove, two velocimeters were used to get the correlations $S_{v v}[y](\boldsymbol{r}, \tau)$. The distance vector between the two velocimeters is $\boldsymbol{r}$. Let $n_{1}(t)$ and $n_{2}(t)$ be the number of particles detected by each velocimeter at time $t$. LDV being a random sampling technique $n_{1}(t) \neq n_{2}(t)$. The acquisition process is stopped when $n_{p}=n_{1}+n_{2}$ reaches a threshold value fixed at 500,000 particles. The associated final time is denoted $t_{f}$. In an ideal configuration, one could expect to have $n_{1}\left(t_{f}\right) \approx n_{2}\left(t_{f}\right) \approx 250,000$. In practice, as velocimeters are technologically different and most importantly as the seeding is inhomogeneous, especially in the lower part of the boundary layer, this equilibrium is never reached. To avoid spurious measurements inducing convergence issues, runs such that $n_{1}\left(t_{f}\right) \leq 200,000$ or $n_{2}\left(t_{f}\right) \leq 200,000$ were thus eliminated.

The final threshold value $n_{p}=500,000$ was chosen by checking the convergence of the computed statistics. Subsets of raw data with different threshold values were extracted. First, $S_{v v}[y](\boldsymbol{r}, \tau)$ was analyzed and distributions along $z$ obtained with several values of $n_{p}$ are plotted 
in figure 2. As $n_{p}$ increases, convergence is observed, the differences with the reference curve for $n_{p}=500,000$ getting smaller. To quantify the convergence process, integral length scales, further analyzed in section 5.2, are now examined.

Figure 3 shows the convergence of the correlation lengths $\Lambda_{x}, \Lambda_{y}^{\mathrm{inf}}, \Lambda_{y}^{\mathrm{sup}}$ and $\Lambda_{z}$ with respect to the threshold value $n_{p}$. The figure presents the results for location $y / \delta=0.5$ but similar conclusions can be drawn for other locations. A minimum of 200, 000 particles per velocimeter allows to reach a good convergence with an error below $5 \%$ on the integral length scales. We note that convergence depends on the considered integral length scale. In particular, in this example where $y / \delta=0.5$, $\Lambda_{y}^{\text {inf }}$ converged late.

\subsection{Repeatability}

The ergodicity hypothesis is invoked to compute flow statistics but it is not strictly verified. In order to justify this hypothesis, $S_{v v}(y)[\boldsymbol{r}, \tau]$ was evaluated using different sets of data having the same number of particles $n_{p}$. The idea behind this process is to compare time averaged to the ensemble averaged quantities. For each dataset obtained with 500, 000 particles, subsets containing 200, 000 particles are extracted using a sliding window. For altitudes $y / \delta=0.5$ and $y^{+}=180$, the latter being located in the logarithmic region, figures $4 \mathrm{a}$ and $4 \mathrm{~b}$ plot the mean normalized correlation function $S_{v v}(z) /\left\langle v^{\prime 2}\right\rangle$ obtained with 25 subsets and the corresponding standard deviations. The time averaged result obtained with $n_{p}=500,000$ particles is drawn for comparison. Even though 25 subsets can be considered as a limited sampling number, figure 4 a clearly shows that both averaging process provide similar results. Similar conclusion are found for other locations and distributions of $S_{v v}[y](\boldsymbol{r}, \tau)$.

For each of the 25 subsets, the integral length scale $\Lambda_{z}$ was computed. The comparison with the result obtained with the full dataset where $n_{p}=500,000$ leads to a relative uncertainty on $\Lambda_{z}$ of $5 \%$. Once applied to all integral length scales and for all locations, this procedure gives an overall uncertainty of $5 \%$. 


\section{ANALYSIS OF THE VELOCITY CORRELATIONS}

\subsection{Mean velocity and Reynolds stresses}

Before analyzing the data sets for the spatial correlations, a focus is made on the one-point one-time correlations. The eight measured mean velocity profiles are presented in figures $5 \mathrm{a}$ and 5b using dimensionless boundary layer variables or wall units. The DNS results of Sillero et al. [9] $\left(\mathcal{R}_{\theta}=6500\right)$ and a $k-\omega$ SST boundary layer calculation $\left(\mathcal{R}_{\theta}=7260\right)$ using the ONERA code CLICET [32] are also drawn for comparisons. $\mathcal{R}_{\theta}=7260$ correspond to case $U_{e}=50 \mathrm{~m} / \mathrm{s}$, discussed in section 5, while the maximum $\mathcal{R}_{\theta}$ value for the DNS computations [9] is $\mathcal{R}_{\theta}=6500$.

The expected self-similarities of the boundary layers in the inner and outer regions are recovered. Figure $5 \mathrm{~b}$ indicates that the MV gives access to the velocity profiles all over the logarithmic region. However the buffer region and the viscous sublayer are almost not described.

Lysak [7], Aupoix [8] and Morilhat et al. [5] models relate $\left\langle v^{\prime 2}\right\rangle$ profile to the wall pressure spectrum. A good estimation of this profile is thus required to deduced the fluctuating wall pressure. Instead of relying on $\left\langle v^{\prime 2}\right\rangle$ profile directly, the model uses an empirical correlation $\left\langle v^{\prime 2}\right\rangle=-\alpha\left\langle u^{\prime} v^{\prime}\right\rangle$ where $\alpha$ is a constant. Lysak used $\alpha=1$ for pipe flows while Aupoix and Morilhat et al. used $\alpha=1.3$ for zero pressure gradient turbulent boundary layers.

Figure 6 presents the evolution of $\alpha$ across the boundary layer thickness for all tested Reynolds numbers. A rather large plateau region is observed for $y^{+} \in\left[10^{2}, 10^{3}\right]$ whatever the Reynolds number is. Even though the value of the plateau slightly depends on $\mathcal{R}_{\theta}$, considering $\alpha=1.3$ appears to be a reasonable mean value for the plateau region over the range $\mathcal{R}_{\theta} \in[2800,10500]$. As expected, the $k-\omega$ SST computation exhibits the same value for $\alpha$ and the results of Sillero et al. confirm the trend. PIV measurements from Meinhart and Adrian [26] also exhibits a similar plateau for $\mathcal{R}_{\theta} \in[930,6845]$ but the plateau level is slightly higher with $\alpha \approx 1.6$. Differences may be due to the sensitity of PIV to velocity fluctuations levels but the existence of the plateau region is confirmed.

The plateau corresponds to the logarithmic region of the boundary layer, where the LMA model [5] requires the value of $\alpha$ to assess the turbulent contribution to the wall pressure spectrum $\Phi_{p p}$. 
Below the logarithmic region, the contribution of $\Phi_{P}$ in eq. (2) is low because $\left\langle v^{\prime 2}\right\rangle$ tends to zero and most importantly because of the term $l_{e}^{5}$. Above the logarithmic region $\left\langle v^{\prime 2}\right\rangle$ quickly decreases and the main contribution to $\Phi_{p p}$ is due to the mean velocity profile [5], the turbulent contribution due to $\left\langle v^{\prime 2}\right\rangle$ being negligible. Finally, considering $\alpha=1.3$ all across the boundary layer and independent of the Reynolds number is a reasonable approximation for the LMA model.

\subsection{Integral length scales}

Two-point spatial correlations are now examined. The external velocity is $U_{e}=50 \mathrm{~m} / \mathrm{s}$ leading to $\mathcal{R}_{\theta}=7260$. The time delay $\tau$ is fixed at zero and is removed from the variable list of $S_{v v}$. For each of the measured correlations $S_{v v}(\mathbf{r})$, for which the distance vector $\mathbf{r}$ is aligned with one of the directions $x, y$ or $z$, the integral length scale $\Lambda$ is calculated as the area of the normalized correlations function $S_{v v} /\left\langle v^{\prime 2}\right\rangle$ :

$$
\Lambda_{i}=\int_{0}^{\infty} \frac{S_{v v}\left(r_{i} \mathbf{e}_{\mathbf{i}}\right)}{\left\langle v^{\prime 2}\right\rangle} d r_{i}
$$

$S_{v v}$ and $\left\langle v^{\prime 2}\right\rangle$ implicitly depend on the altitude $y_{0}$, thus $\Lambda_{i}$ is a function of $y_{0}$.

Figures $7 \mathrm{a}$ to $7 \mathrm{f}$ show the three 1D normalized correlations, plotted with respect to the distance $r_{i}$ normalized by the respective integral length scales $\Lambda_{i}$. DNS results of Sillero were analyzed similarly and are drawn for comparison purpose. Concerning the wall-normal direction $y$, two distinct length scales are computed. $\Lambda_{y}^{\text {sup }}$ refers to the evolution of the correlation for positive values of the distance $r_{y}$, while $\Lambda_{y}^{\mathrm{inf}}$ stands for the negative distance toward the wall. Non-physical

behaviors are observed on the DNS for the normalized correlations functions $\frac{S_{v v}\left(0, r_{y}, 0\right)}{\left\langle v^{\prime 2}\right\rangle}$ for high values of $y_{0}$ when $r_{y}$ increases. This is due to the boundary condition in the DNS of Sillero et al. placed at $2 \delta$ and where $v^{\prime}$ fluctuations are set to zero.

On each of these figures, the associated Von Kármán isotropic correlation function $S_{v v}^{V K}$ is also depicted. This function corresponds to the turbulence filter $\Phi_{T}$ involved in the LMA (2) model. The function is computed in the following manner. For two-point one-time correlation, $S_{v v}(\mathbf{r})$ is deduced from the wave-number spectrum $\Phi_{v v}(\mathbf{k})$ through an inverse Fourier transform: 


$$
S_{v v}(\mathbf{r})=\frac{1}{(2 \pi)^{3}} \int_{\mathbb{R}^{3}} \Phi_{v v}(\mathbf{k}) e^{j \mathbf{k} \cdot \mathbf{r}} d \mathbf{k}
$$

In homogeneous isotropic turbulence, the velocity spectrum is related to the energy spectrum through:

$$
\Phi_{v v}(\mathbf{k})=\frac{E(\mathbf{k})}{4 \pi k^{2}}\left(1-\frac{k_{y}^{2}}{k^{2}}\right)
$$

where $k=\|\mathbf{k}\|$.

The energy spectrum for isotropic turbulence may be approximated by the Von Kármán model:

$$
E(\mathbf{k}) \propto \frac{\left\langle v^{\prime 2}\right\rangle}{k_{e}} \frac{\left(\frac{k}{k_{e}}\right)^{4}}{\left(1+\left(\frac{k}{k_{e}}\right)^{2}\right)^{\frac{17}{6}}}
$$

where $k_{e}=1 / l_{e}$ is the characteristic wavenumber carrying the maximum of energy.

For 1D correlations, the integral in eq.(4) simplifies and analytical solutions are achieved. For the normalized correlations such that $S_{v v}^{V K}(\mathbf{0})=\left\langle v^{\prime 2}\right\rangle$, they read: 


$$
\begin{aligned}
S_{v v}^{V K}\left(r_{x}, 0,0\right) & \propto\left(r_{x} k_{e}\right)^{1 / 3}\left(2 K_{1 / 3}\left(r_{x} k_{e}\right)\right. \\
& \left.-r_{x} k_{e} K_{2 / 3}\left(r_{x} k_{e}\right)\right) \\
S_{v v}^{V K}\left(0,0, r_{z}\right) & \propto\left(r_{z} k_{e}\right)^{1 / 3}\left(2 K_{1 / 3}\left(r_{z} k_{e}\right)\right. \\
& \left.-r_{z} k_{e} K_{2 / 3}\left(r_{z} k_{e}\right)\right) \\
S_{v v}^{V K}\left(0, r_{y}, 0\right) & \propto\left(r_{y} k_{e}\right)^{1 / 3} K_{1 / 3}\left(r_{y} k_{e}\right)
\end{aligned}
$$

where $K_{1 / 3}$ and $K_{2 / 3}$ are modified Bessel functions of the second kind. Functions in eq. (7) stand for the 1D correlations in an equivalent isotropic flow. Deviations from these functions reveal anisotropic effects.

A more general analytical form for $S_{v v}^{V K}\left(r_{x}, r_{y}, r_{z}\right)$ is also accessible:

$$
\begin{aligned}
S_{v v}^{V K}\left(r_{x}, r_{y}, r_{z}\right) & \propto 2\left(k_{e} r\right)^{1 / 3} \mathrm{~K}_{1 / 3}\left(k_{e} r\right) \\
& -k_{e}^{4 / 3}\left(r_{x}^{2}+r_{z}^{2}\right) r^{-2 / 3} \mathrm{~K}_{2 / 3}\left(k_{e} r\right)
\end{aligned}
$$

where $r=\|\mathbf{r}\|$.

Figures $7 \mathrm{a}$ to $7 f$ show a good collapse of all the correlations with a weak influence of the altitude $y_{0}$, indicating that the Von Kármán correlation function (7) is a satisfactory approximation of decay of the normalized correlation functions. It is observed in the experiments and in the DNS that departures from isotropic behaviors evolve reversely with respect to the altitude $y_{0}$ between $S_{v v}\left(r_{x}, 0,0\right)$ and $S_{v v}\left(0,0, r_{z}\right)$. Furthermore, in figures $7 \mathrm{e}$ and $7 \mathrm{f}$ curves do not collapse for negative values of $r_{y}$ with the normalization using $\Lambda_{y}^{\text {sup }}$. However, with a normalization based on $\Lambda_{y}^{\inf }$ a good collapse is observed. 
The four length scales are plotted with respect to the altitude $y_{0}$ in the boundary layer on figure 8. Results obtained in the present experiments are consistent with those found by Sillero et al. [9] and used by Slama et al. [3], even though some discrepancies exist for $\Lambda_{x}$ and $\Lambda_{y}^{\text {sup }}$. Below $y=0.3 \delta$, the behavior of $\Lambda_{x}$ observed in the DNS departs from the one measured in the present study. More surprisingly, in the inner region of the boundary layer for $y<0.2 \delta$ and in the outer region $y>0.5 \delta$, experimental results for $\Lambda_{y}^{\text {sup }}$ show behaviors in complete disagreement with DNS data. In the outer region, the boundary condition used in the DNS is suspected to generate the strong increase of $\Lambda_{y}^{\text {sup }}$, as already pointed out in figure $7 \mathrm{f}$. But so far, there is no explanation to explain the observed behaviors of the DNS and the measurements in the inner region.

\subsection{Convection velocity and decorrelation time scale}

The velocity correlations are now used to extract the convection velocity $U_{c}$ at which the fluctuations are advected and the associated time scale $\tau_{S}$. Considering that the convection is essentially longitudinal, only $S_{v v}\left(r_{x}, 0,0, \tau\right)$ is scrutinized. For $y_{0}=0.18 \delta$, time evolution of the correlations $S_{v v}\left(r_{x}, 0,0, \tau\right)$ are shown for five increasing values of the distance $r_{x}$ on figure 9 . This figure illustrates the transport of the correlations with the dissipation and the dispersion of $S_{v v}\left(r_{x}, 0,0, \tau\right)$ as $r_{x}$ increases.

Experimentally [33] in boundary layer flows, the correlation $S_{u u}\left(0, r_{y}, 0, \tau\right)$ was found to present maxima positively or negatively delayed with respect to the sign of $r_{y}$. This is due to inclined and elongated structures in the boundary layers. There are no similar experimental results for $S_{v v}\left(0, r_{y}, 0, \tau\right)$. From the DNS results of Sillero et al. [9], Slama et al. [3] shows that $S_{v v}\left(0, r_{y}, 0, \tau\right)$ exhibits no inclination, the iso-contours of $S_{v v}\left(r_{x}, r_{y}, 0,0\right)$ for $r_{x}=0$ being almost vertically aligned, unlike $S_{u u}\left(r_{x}, r_{y}, 0,0\right)$ or $S_{w w}\left(r_{x}, r_{y}, 0,0\right)$. The present measurements confirm this trend as shown in figure 10. No time delays are observed on $S_{v v}\left(0, r_{y}, 0, \tau\right)$. The correlation simply decreases and slightly widens as $\left|r_{y}\right|$ increases.

The complete evolution with respect to the normalized delay $\tau U_{e} / \delta$ of $S_{v v}\left(r_{x}, 0,0, \tau\right)$ is presented in figure 11a for all the acquired longitudinal distance $r_{x}$. Adjusting an exponential decay $e^{-\tau / \tau_{S}}$ as illustrated on the figure allows to evaluate the decorrelation time scale $\tau_{S}$. A similar approach was already used by Tam et al. [34] and Ewert et al. [35,36] to represent the decorrelation 
of the velocity fluctuations with time. Time scale $\tau_{S}$ is plotted in figure $11 \mathrm{~b}$ for all altitude $y_{0}$. The decorrelation time scale increases across the boundary layer suggesting that the convection velocity increases accordingly. The model used by Morilhat et al. [5] is also depicted in figure 11b. A very satisfactory agreement is observed validating the approach [34,35]. In addition, the exponential decay observed on figure 11a justify the form of the advection filter $\Phi_{A}$ of the LMA model (2).

To evaluate the convection velocity $U_{c}$, the peak value of $S_{v v}\left(r_{x}, 0,0, \tau\right)$ is extracted for each distance $r_{x}$ on figure 11a. Applying this procedure to all altitude $y_{0}$ in the boundary layer leads to figure 12a. For each altitude $y_{0}$, the peak value of $S_{v v}\left(r_{x}, 0,0, \tau\right)$ has a quasi linear evolution in the $\left(r_{x}, \tau\right)$ plane suggesting that the convection velocity is constant. Furthermore, the convection velocity is always lower that $U_{e}$ (blue line) and depends on $y_{0}$. A linear regression provides an estimate of $U_{c}$ and its variation throughout the boundary layer is shown on figure $12 \mathrm{~b}$. The convection velocity increases with $y_{0}$ and is very similar to the mean velocity $U$ in the outer region of the boundary layer. This is consitent with the results obtained by [37] by means of DNS. Again, the use of Taylor's hypothesis for the transport of the wall-normal velocity fluctuations in the LMA model is strengthen by these experimental results showing that $U_{c}$ is very close to $U$.

\section{CONCLUSIONS}

A measurement campaign was performed to describe the spatial correlations of the wallnormal velocity in a zero pressure gradient turbulent boundary layer. Two LDV systems were employed to access the velocity correlations. These measurements complement the existing experimental and numerical database $([9,14,15])$ by providing spatial correlations for the wall-normal velocity. A unique data-set was obtained that should help improving the recent modeling efforts focused on wall pressure fluctuations $([3,5])$.

The ratio $\alpha=-\left\langle v^{\prime 2}\right\rangle /\left\langle u^{\prime} v^{\prime}\right\rangle$ is found to be around 1.3 in the logarithmic region of the boundary layer, irrespective of the Reynolds number in the range $\mathcal{R}_{\theta} \in[2800,10500]$. This result confirms previous studies for which the same value of $\alpha$ was also found. More interestingly, the spatial damping of the correlation function $S_{v v}\left(r_{i} \mathbf{e}_{\mathbf{i}}, 0\right)$ may be reasonably approximated using a model 
based on the Von Kármán spectrum for $\Phi_{v v}(\mathbf{k})$. The only remaining unknown parameters are the length scales $\Lambda_{i}$ that may be modeled from the results obtained in this study for instance. Furthermore, the decorrelation time scale $\tau_{S}$ is proved to increase across the boundary layer thickness proportionally to $1 / \omega_{t}=C_{\mu} k_{t} / \varepsilon_{t}$. Additionally, the measured convection velocity $U_{c}$ of the $S_{v v}$ correlations is found to be very close to the mean velocity. These experimental results thus confirm that the use of Taylor frozen turbulence hypothesis for $S_{v v}$ is justified to model $\Phi_{p p}(\mathbf{k}, \omega)$.

Concerning the LMA model, further developments are required to account for the anisotropy of the turbulent structures carrying wall-normal fluctuations. While a unique length scale based on $k_{e}$ acts in the actual LMA model, different integral length scales $\Lambda_{i}$ should be accounted for in the future model. This should improve the prediction of the wall pressure spectrum amplitude and its frequency distribution. From the experimental side, a complementary experimental campaign focused on wall pressure spectra in the same configuration would be of major interest to assess the LMA model regarding $\Phi_{p p}$. The present wind-tunnel was too noisy to perform accurate measurements of wall pressure fluctuations and, for the sake of consistency, it would be interesting to acquire a complete set of velocity and pressure correlations in an aeroacoustic wind tunnel. 


\section{NOMENCLATURE}

$U_{e}$ freestream velocity

$U_{c}$ convection velocity

$U$ longitudinal velocity component

$v$ wall-normal velocity component

$x$ longitudinal direction

y wall-normal direction

$z \quad$ transverse direction

$t$ time

$\mathbf{x}$ position vector $(x, y, z)$

r displacement vector

k wave number vector $\left(k_{x}, k_{y}, k_{z}\right)$

$\omega$ angular frequency

$\tau_{S}$ decorrelation time scale

$\Lambda_{i} \quad$ integral length scale in the direction $i$

$S_{q q} \quad$ correlation function for quantity $q$

$\rho$ density

$\nu \quad$ kinematic viscosity

$\tau_{w} \quad$ wall shear stress

$\mathcal{R}_{\theta} \quad$ Reynolds number based on the momentum thickness

$k_{t} \quad$ turbulent kinetic energy

$\varepsilon_{t} \quad$ turbulent dissipation

$\omega_{t}$ turbulent specific dissipation

+ superscript for dimensionless wall quantities

' superscript for fluctuating quantities

〈〉 Reynolds average 


\section{REFERENCES}

[1] Kraichnan, R. H., 1956. "Pressure fluctuations in turbulent flow over a flat plate". The Journal of the Acoustical Society of America, 28(3), pp. 378-390.

[2] Panton, R. L., and Linebarger, J. H., 1974. "Wall pressure spectra calculations for equilibrium boundary layers". Journal of Fluid Mechanics, 65(2), pp. 261-287.

[3] Slama, M., Leblond, C., and Sagaut, P., 2018. "A kriging-based elliptic extended anisotropic model for the turbulent boundary layer wall pressure spectrum". Journal of Fluid Mechanics, 840, pp. 25-55.

[4] Peltier, L. J., and Hambric, S. A., 2007. "Estimating turbulent-boundary-layer wall-pressure spectra from cfd rans solutions". Journal of Fluids and Structures, 23(6), pp. 920-937.

[5] Morilhat, S., Chedevergne, F., and Simon, F., 2019. "A unified methodology to evaluate the radiated noise due to turbulent boundary layer flows". ASME Journal of Fluids Engineering, 141(6), dec, p. 061201.

[6] Morilhat, S., 2018. "Modélisation des fluctuations de la pression pariétale d'une couche limite turbulente pour des applications en vibro-acoustique". PhD thesis, Université de Toulouse.

[7] Lysak, P. D., 2006. "Modeling the wall pressure spectrum in turbulent pipe flows". Journal of fluids engineering, 128(2), pp. 216-222.

[8] Aupoix, B., 2015. "Extension of Lysak s Approach to Evaluate the Wall Pressure Spectrum for Boundary Layer Flows". Flow, Turbulence and Combustion, 94(1), pp. 63-78.

[9] Sillero, J., Jimenez, J., and Moser, R., 2014. "Two-point statistics for turbulent boundary layers and channels at reynolds numbers up to $\delta^{+}=2000$ ". Physics of fluids, 26(105109).

[10] Blake, W. K., 1970. "Turbulent boundary-layer wall-pressure fluctuations on smooth and rough walls". Journal of Fluid Mechanics, 44(04), pp. 637-660.

[11] McGrath, B. E., and Simpson, R. L., 1987. "Some features of surface pressure fluctuations in turbulent boundary layers with zero and favorable pressure gradients".

[12] Goody, M. C., and Simpson, R. L., 2000. "Surface pressure fluctuations beneath two-and three-dimensional turbulent boundary layers". AIAA journal, 38(10), pp. 1822-1831.

[13] Arguillat, B., 2006. "Etude expérimentale et numérique de champs de pression pariétale dans 
lespace des nombres donde, avec application aux vitrages automobiles". PhD thesis, Ecole centrale de Lyon.

[14] Favre, A., Gaviglio, J., and Dumas, R., 1957. "Space-time double correlations and spectra in a turbulent boundary layer". Journal of Fluid Mechanics, 2(4), pp. 313-342.

[15] Tritton, D., 1967. "Some new correlation measurements in a turbulent boundary layer". Journal of Fluid Mechanics, 28(3), pp. 439-462.

[16] Grant, H., 1958. "The large eddies of turbulent motion". Journal of Fluid Mechanics, 4(2), pp. 149-190.

[17] Belmabrouk, H., and Michard, M., 1998. "Taylor length scale measurement by laser doppler velocimetry". Experiments in Fluids, 25(1), pp. 69-76.

[18] Neumann, M., Heitkam, S., Shirai, K., Büttner, L., and Czarske, J. "Highly precise correlation estimates of turbulent shear flows using a laser doppler profile sensor and array detection".

[19] Fraser, R., Pack, C., and Santavicca, D., 1986. "An Idv system for turbulence length scale measurements". Experiments in fluids, 4(3), pp. 150-152.

[20] Bech, K. H., Tillmark, N., Alfredsson, P. H., and Andersson, H. I., 1995. "An investigation of turbulent plane couette flow at low reynolds numbers". Journal of Fluid Mechanics, 286, pp. 291-325.

[21] Furuichi, N., Hachiga, T., and Kumada, M., 2004. "An experimental investigation of a largescale structure of a two-dimensional backward-facing step by using advanced multi-point ldv". Experiments in Fluids, 36(2), pp. 274-281.

[22] Benedict, L., and Gould, R., 1999. "Understanding biases in the near-field region of Ida two-point correlation measurements”. Experiments in fluids, 26(5), pp. 381-388.

[23] Trimis, D., and Melling, A., 1995. "Improved laser doppler anemometry techniques for twopoint turbulent flow correlations". Measurement Science and Technology, 6(6), p. 663.

[24] Ölçmen, M. S., Simpson, R. L., and Goody, M., 2001. "An experimental investigation of twopoint correlations in two-and three-dimensional turbulent boundary layers". Flow, turbulence and combustion, 66(2), pp. 85-112.

[25] Violato, D., and Scarano, F., 2011. "Three-dimensional evolution of flow structures in transi- 
tional circular and chevron jets". Physics of Fluids, 23(12), p. 124104.

[26] Meinhart, C., and Adrian, R., 1995. "Measurement of the zero-pressure gradient turbulent boundary layer using particle image velocimetry". In 33rd Aerospace Sciences Meeting and Exhibit, p. 789.

[27] Kähler, C., 2004. "Investigation of the spatio-temporal flow structure in the buffer region of a turbulent boundary layer by means of multiplane stereo piv". Experiments in Fluids, 36(1), pp. 114-130.

[28] Ganapathisubramani, B., Hutchins, N., Hambleton, W., Longmire, E., and Marusic, I., 2005. "Investigation of large-scale coherence in a turbulent boundary layer using two-point correlations". Journal of Fluid Mechanics, 524, pp. 57-80.

[29] Adrian, R. J., Meinhart, C. D., and Tomkins, C. D., 2000. "Vortex organization in the outer region of the turbulent boundary layer". Journal of Fluid Mechanics, 422, pp. 1-54.

[30] Clauser, F., 1956. "The turbulent boundary layer”. In Advances in Applied Mechanics. Elsevier, pp. 1-51.

[31] Wei, T., Schmidt, R., and McMurtry, P., 2005. "Comment on the clauser chart method for determining the friction velocity". Experiments in fluids, 38(5), pp. 695-699.

[32] Aupoix, B., 2015. Clicet - version 2015 descriptif et mode d'emploi. Rapport Technique 1/24088 DMAE, ONERA, Février.

[33] Favre, A., Gaviglio, J., and Dumas, R., 1958. "Further space-time correlations of velocity in a turbulent boundary layer". Journal of Fluid Mechanics, 3(4), pp. 344-356.

[34] Tam, W., Christopher, K., and Auriault, L., 1999. "Jet mixing noise from fine-scale turbulence". AIAA journal, 37(2), pp. 145-153.

[35] Ewert, R., 2008. "Broadband slat noise prediction based on caa and stochastic sound sources from a fast random particle-mesh (rpm) method". Computers \& Fluids, 37(4), pp. 369-387.

[36] Ewert, R., Dierke, J., Siebert, J., Neifeld, A., Appel, C., Siefert, M., and Kornow, O., 2011. "Caa broadband noise prediction for aeroacoustic design". Journal of sound and vibration, 330(17), pp. 4139-4160.

[37] Renard, N., and Deck, S., 2015. "On the scale-dependent turbulent convection velocity in a 
ASME Journal of Fluids Engineering

spatially developing flat plate turbulent boundary layer at reynolds number". Journal of Fluid Mechanics, 775, jun, pp. 105-148. 


\section{LIST OF FIGURES}

1 Picture of the wind tunnel test section equipped with the two LDV benches. The "blue" LDV bench is on the left hand side while the "yellow" one is on the right, behind the test section. . . . . . . . . . . . . . . . . . 26

2 Convergence of the correlation function $S_{v v}[y](z)$ with respect to $n_{p} \in[20,000 ; 400,000]$ for $y / \delta=0.5$. The dashed line corresponds to full raw data with $n_{p}=500,000 \ldots$. . 27

3 Convergence of correlation length scales with respect to the number of acquired particles for $y / \delta=0.5 . \Lambda_{n_{p}}$ is the value of the correlation length scale computed with $n_{p}$ particles while $\Lambda_{n_{\max }}$ is the retained value of the correlation length scale obtained with $n_{\max }=500,000 \ldots \ldots \ldots \ldots \ldots \ldots \ldots$

4 Mean and standard deviation of 25 extracted subsets compared with full dataset. . . 29

5 Mean longitudinal velocity profiles obtained for $\mathcal{R}_{\theta} \in[2800,10500]$. Gray to black colors indicate increasing $\mathcal{R}_{\theta}$ values. (a) the normalization is based on the outer scales $\delta$ and $U_{e}$. (b) the normalization lies on the wall variables $y^{+}=\frac{y u_{\tau}}{\nu}$ and $u_{\tau}=\sqrt{\frac{\tau_{w}}{\rho}}$. The solid line $(-)$ is the DNS results of Sillero et al. at $\mathcal{R}_{\theta}=6500$ and the dashed line $(--)$ is the $k-\omega$ SST results at $\mathcal{R}_{\theta}=7260 \ldots \ldots$. . . . . . 30

6 Ratio $\alpha=-\left\langle v^{\prime 2}\right\rangle /\left\langle u^{\prime} v^{\prime}\right\rangle$ with respect to $y^{+}$for $\mathcal{R}_{\theta} \in[2800,10500]$. Gray to black colors indicate increasing $\mathcal{R}_{\theta}$ values. The blues lines show Sillero et al. DNS results $(-)$ and $k-\omega$ SST computation (- - ).

7 Spatial correlations of the wall-normal velocity $S_{v v}$ obtained for $\mathcal{R}_{\theta}=7260$. On the left hand side, LDV measurements are plotted using symbols, while on the right hand side, DNS results of Sillero et al. are drawn with solid lines. Gray to black colors indicate increasing $y_{0}$ values. Blue lines correspond to the Von Kármán correlation model of eq. (7). . . . . . . . . . . . . . . . . . . . . 32

8 Integral length-scale evolutions across the boundary layer, $\Lambda_{x}$ : ० symbol, $\Lambda_{y}^{\text {sup }}$ : symbol, $\Lambda_{y}^{\mathrm{inf}}:+$ symbol and $\Lambda_{z}: \times$ symbol. Symbols present LDV measurements and lines with symbols stand for DNS results. . . . . . . . . . . . . . . . . . . 33 
9 Transport of the velocity correlation. For each value of the distance vector $\mathbf{r}=$ $\left(r_{x}, 0,0\right)$, the normalized velocity correlation $S_{v v}\left(r_{x}, 0,0, \tau\right) /\left\langle v^{\prime 2}\right\rangle$ is plotted with respect to $\tau$. The altitude is fixed at $y_{0} / \delta=0.18 \ldots \ldots \ldots \ldots \ldots$

10 Normal distribution of the velocity correlation. For each value of the distance vector $\mathbf{r}=\left(0, r_{y}, 0\right)$, the normalized velocity correlation $S_{v v}\left(0, r_{y}, 0, \tau\right) /\left\langle v^{\prime 2}\right\rangle$ is plotted with respect to $\tau$. The altitude is fixed at $y_{0} / \delta=0.18 \ldots \ldots \ldots \ldots \ldots$

11 Decorrelation time scale. (a) evolution of $S_{v} v\left(r_{x}, 0,0, \tau\right)$ with respect to $\tau$. Black to gray colors indicate increasing $r_{x}$ values. Velocity correlations are obtained for $y_{0} / \delta=0.18, \mathcal{R}_{\theta}=7260$. (b) Profile of the decorrelation time scale $\tau_{S}$ in the boundary

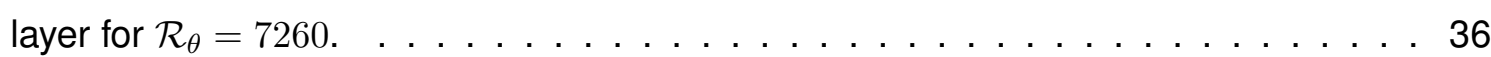

12 Convection of the wall-normal velocity correlations. (a) evolution of the peak values of $S_{v v}\left(r_{x}, 0,0, \tau\right)$ in the plane $\left(r_{x}, \tau\right)$. Gray to black colors indicate increasing $y_{0}$ values. The blue line represents the linear convection at constant speed $U_{e}$. (b) Profile of the convection velocity $U_{c}$ ( ( symbols) compared to the mean longitudinal velocity profile $(+$ symbols $) . \ldots \ldots$. . . . . . . . . . . . . . . . 37 


\section{LIST OF TABLES}

1 Characteristics of the velocimeters $\ldots \ldots \ldots \ldots$

2 Characteristics of the measured boundary layers . . . . . . . . . . . . . 39

3 Maxima values used for the vector distance $\mathbf{r}$ in each direction for one-dimensional or bi-dimensionnal explorations . . . . . . . . . . . . . . . . . . . 40

$4 \quad$ Number of spatial correlations acquired for each kind of measurements and the associated data volumes . . . . . . . . . . . . . . . . . . . . . . 41 


\section{FIGURES}

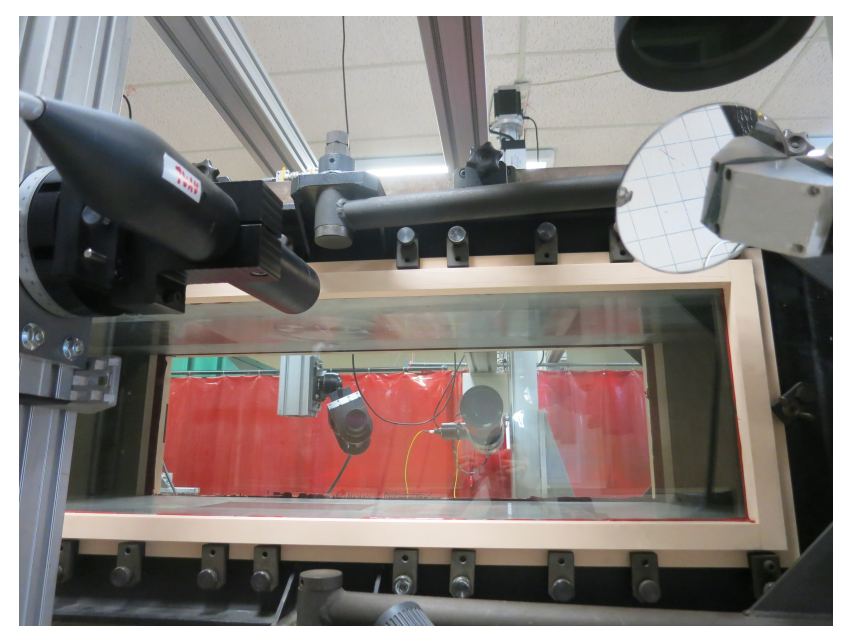

Fig. 1: Picture of the wind tunnel test section equipped with the two LDV benches. The "blue" LDV bench is on the left hand side while the "yellow" one is on the right, behind the test section. 


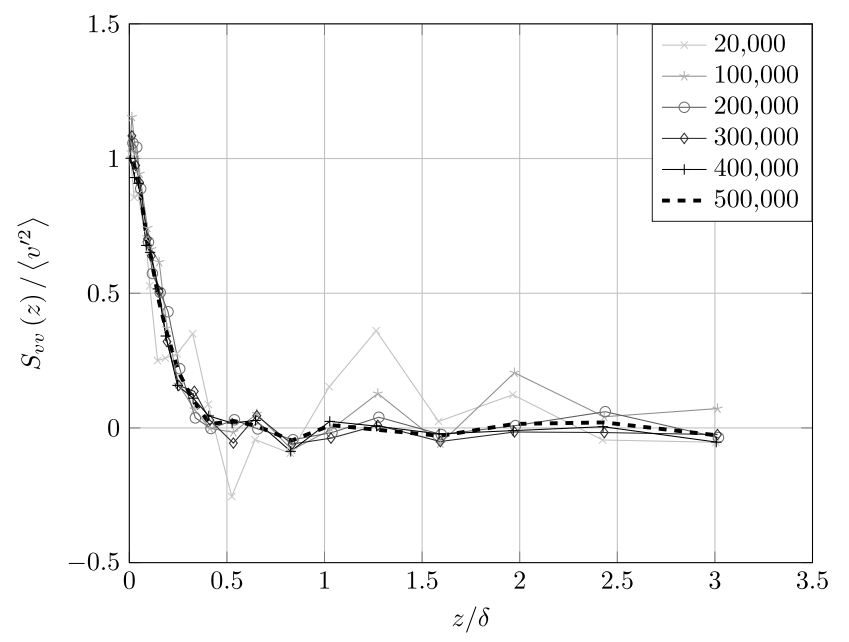

Fig. 2: Convergence of the correlation function $S_{v v}[y](z)$ with respect to $n_{p} \in[20,000 ; 400,000]$ for $y / \delta=0.5$. The dashed line corresponds to full raw data with $n_{p}=500,000$. 


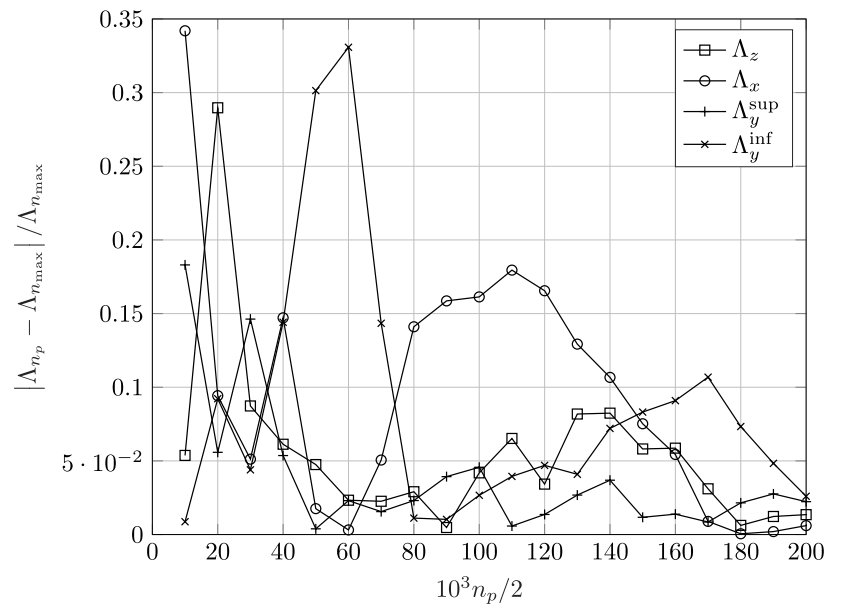

Fig. 3: Convergence of correlation length scales with respect to the number of acquired particles for $y / \delta=0.5 . \Lambda_{n_{p}}$ is the value of the correlation length scale computed with $n_{p}$ particles while $\Lambda_{n_{\max }}$ is the retained value of the correlation length scale obtained with $n_{\max }=500,000$. 


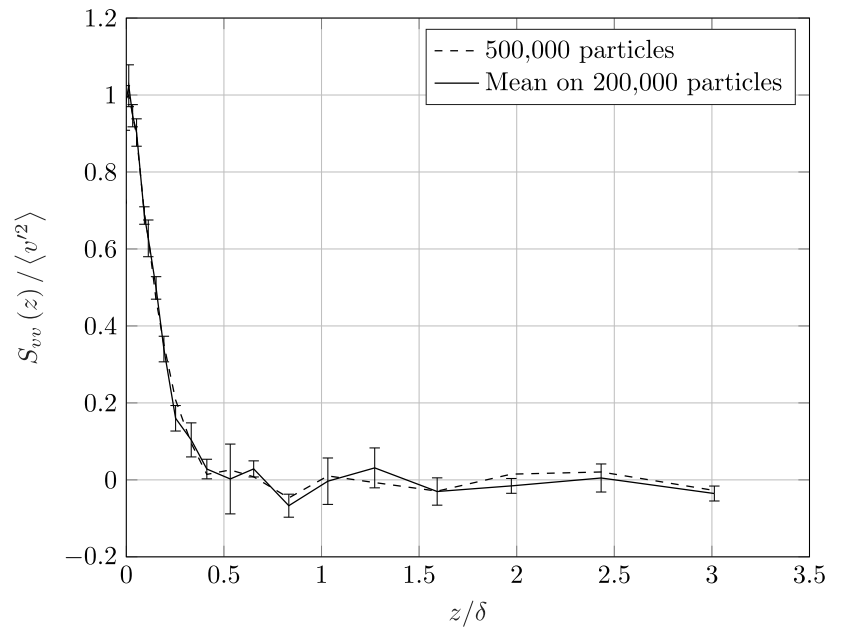

(a) $y / \delta=0.5$

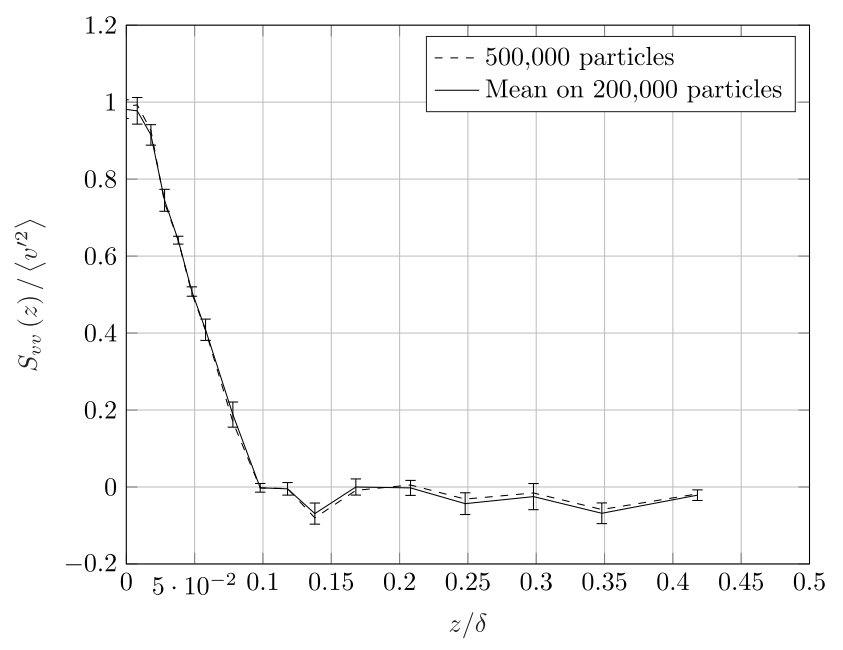

(b) $y^{+}=180$

Fig. 4: Mean and standard deviation of 25 extracted subsets compared with full dataset. 


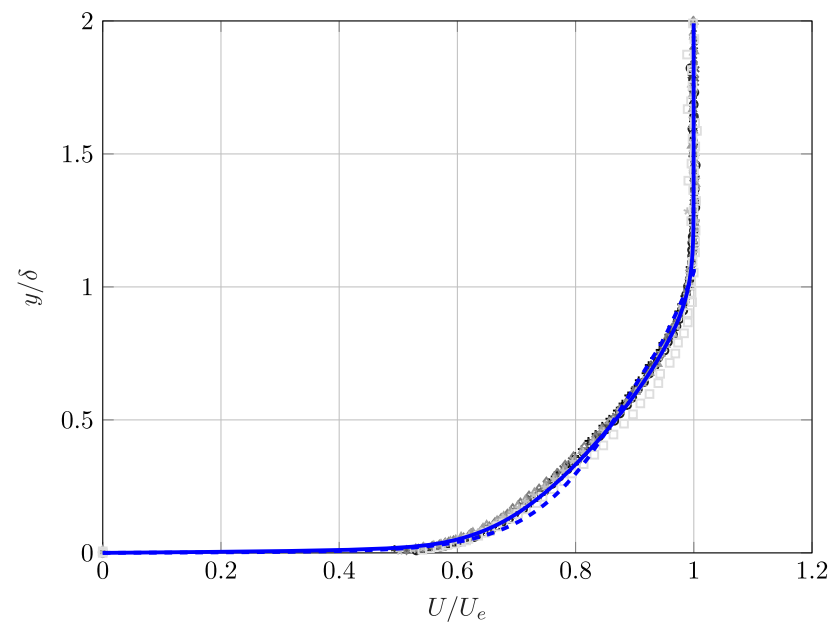

(a) Outer region scaling

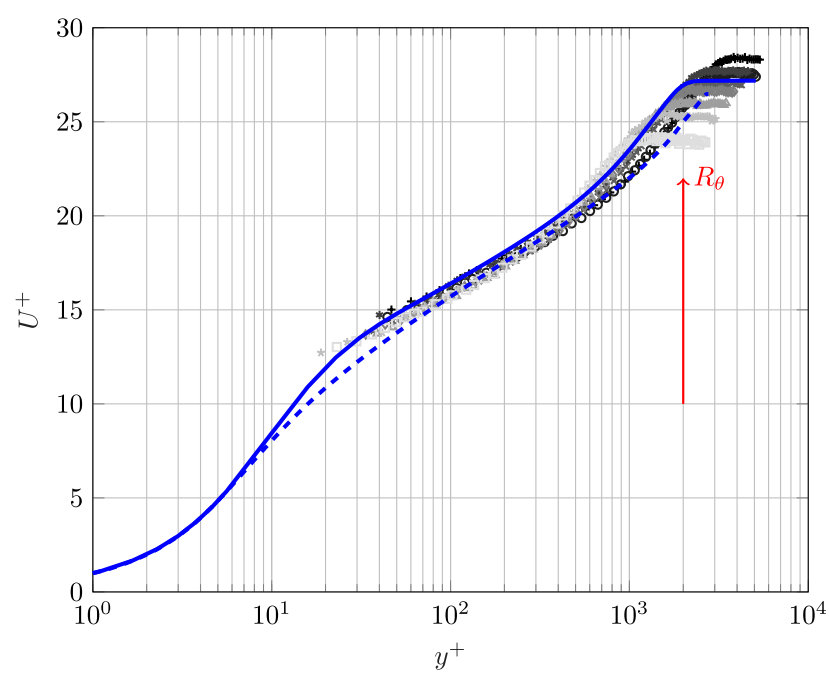

(b) Inner region scaling

Fig. 5: Mean longitudinal velocity profiles obtained for $\mathcal{R}_{\theta} \in[2800,10500]$. Gray to black colors indicate increasing $\mathcal{R}_{\theta}$ values. (a) the normalization is based on the outer scales $\delta$ and $U_{e}$. (b) the normalization lies on the wall variables $y^{+}=\frac{y u_{\tau}}{\nu}$ and $u_{\tau}=\sqrt{\frac{\tau_{w}}{\rho}}$. The solid line $(-)$ is the DNS results of Sillero et al. at $\mathcal{R}_{\theta}=6500$ and the dashed line (- - ) is the $k-\omega$ SST results at $\mathcal{R}_{\theta}=7260$. 


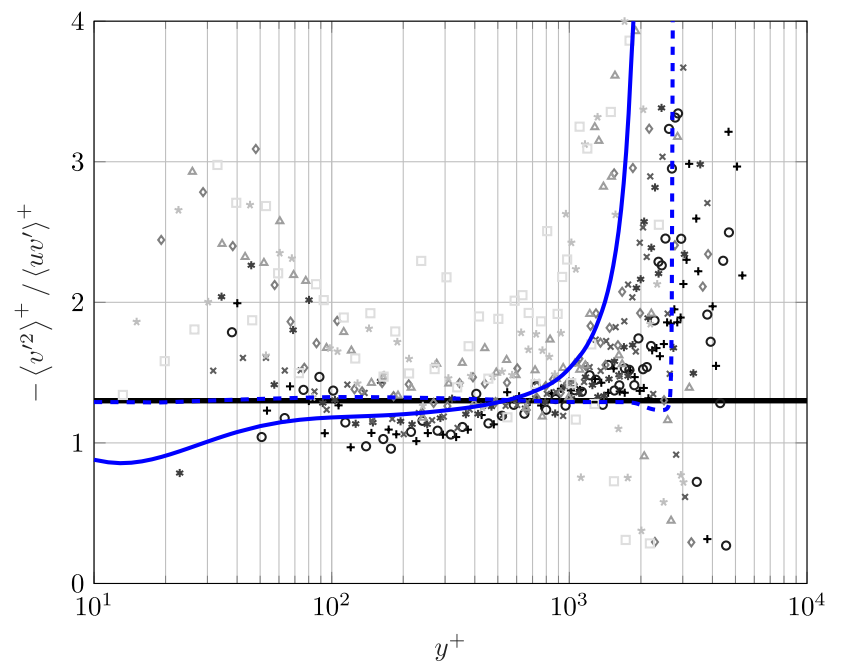

Fig. 6: Ratio $\alpha=-\left\langle v^{\prime 2}\right\rangle /\left\langle u^{\prime} v^{\prime}\right\rangle$ with respect to $y^{+}$for $\mathcal{R}_{\theta} \in[2800,10500]$. Gray to black colors indicate increasing $\mathcal{R}_{\theta}$ values. The blues lines show Sillero et al. DNS results (-) and $k-\omega$ SST computation (- - -). 


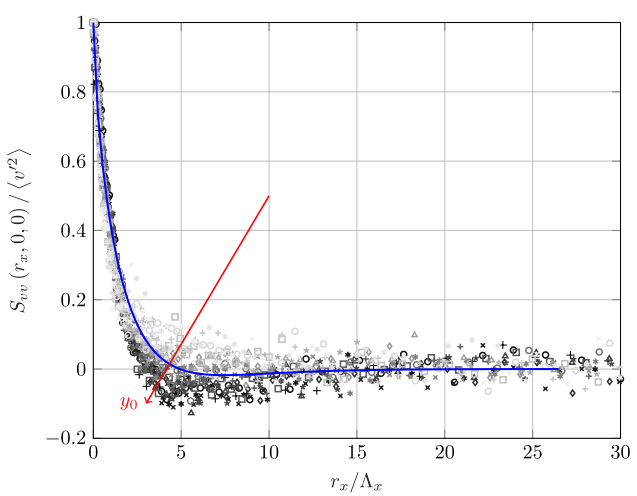

(a) Longitudinal correlation

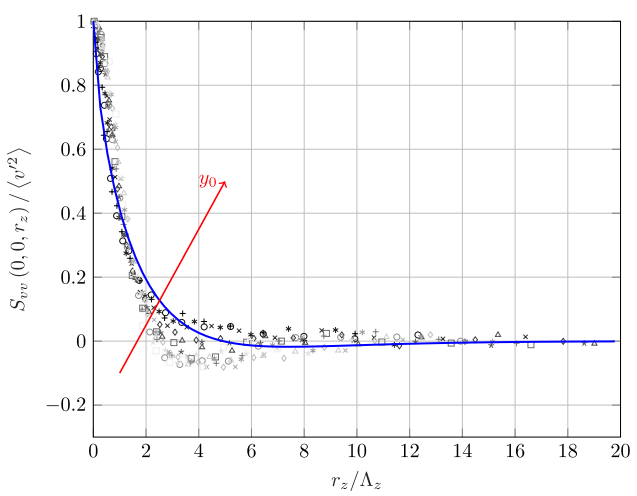

(c) Transverse correlation

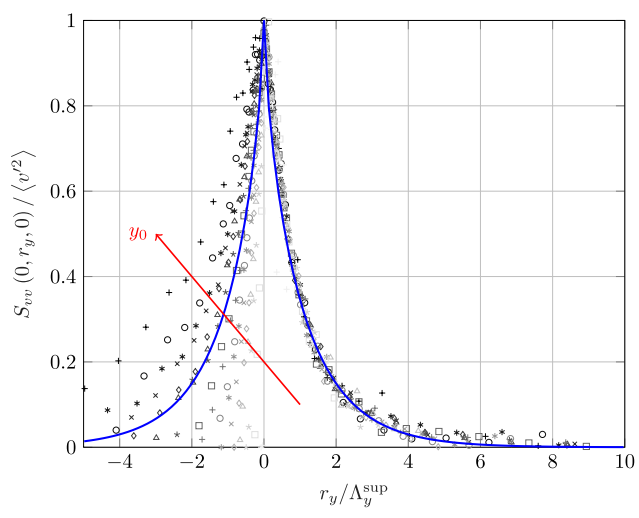

(e) Normal correlation

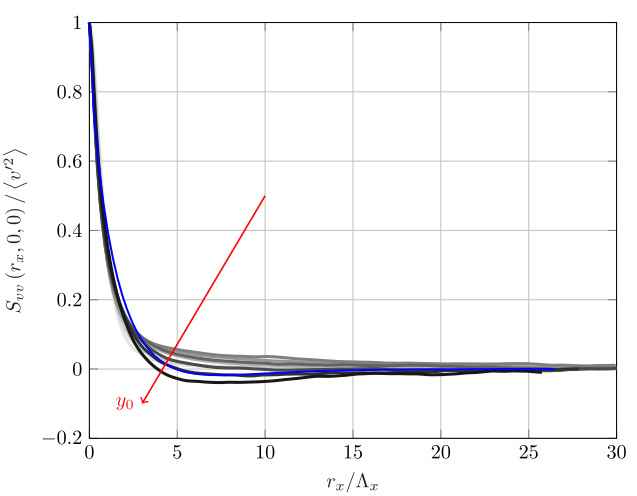

(b) Longitudinal correlation

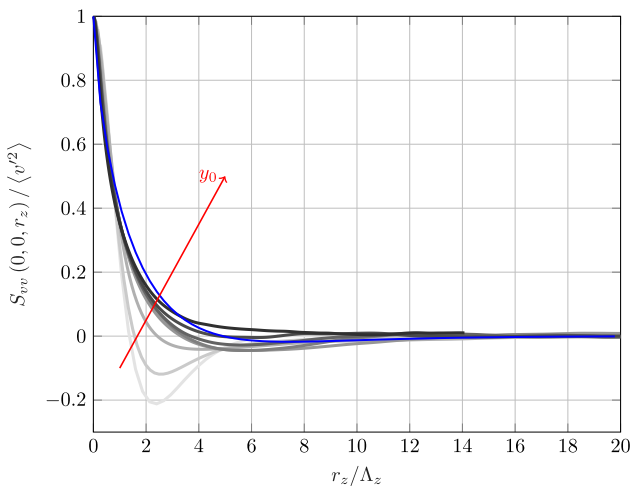

(d) Transverse correlation

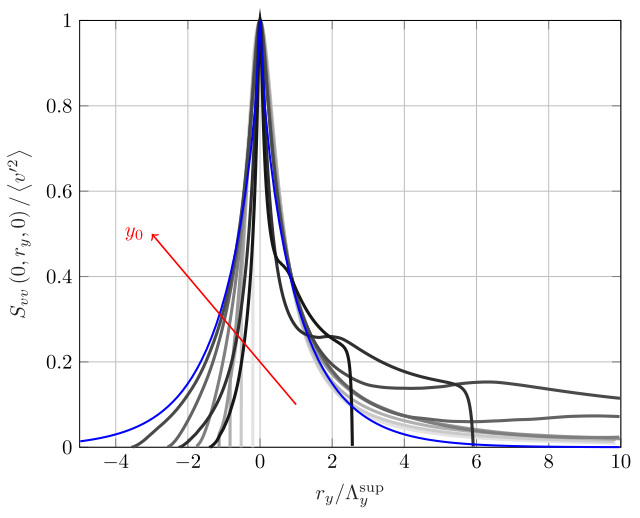

(f) Normal correlation

Fig. 7: Spatial correlations of the wall-normal velocity $S_{v v}$ obtained for $\mathcal{R}_{\theta}=7260$. On the left hand side, LDV measurements are plotted using symbols, while on the right hand side, DNS results of Sillero et al. are drawn with solid lines. Gray to black colors indicate increasing $y_{0}$ values. Blue lines correspond to the Von Kármán correlation model of eq. (7). 


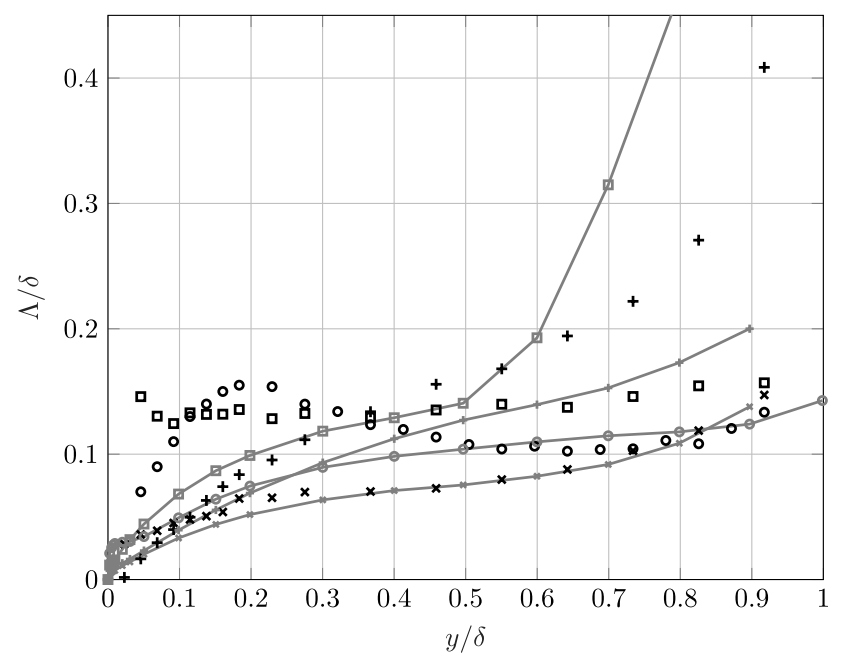

Fig. 8: Integral length-scale evolutions across the boundary layer, $\Lambda_{x}$ : ० symbol, $\Lambda_{y}^{\text {sup }}: \square$ symbol, $\Lambda_{y}^{\text {inf }}:+$ symbol and $\Lambda_{z}: \times$ symbol. Symbols present LDV measurements and lines with symbols stand for DNS results. 


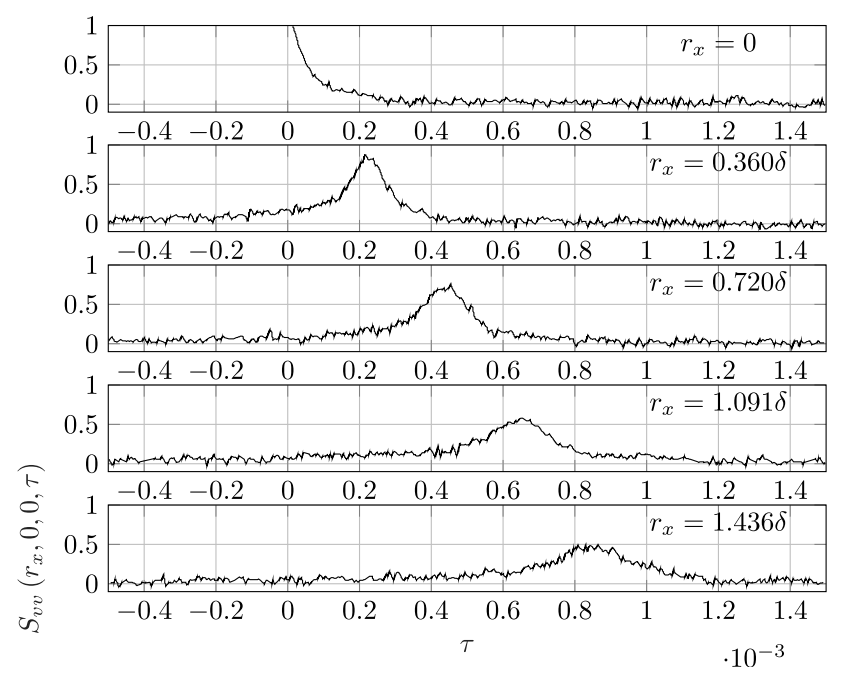

Fig. 9: Transport of the velocity correlation. For each value of the distance vector $\mathbf{r}=\left(r_{x}, 0,0\right)$, the normalized velocity correlation $S_{v v}\left(r_{x}, 0,0, \tau\right) /\left\langle v^{\prime 2}\right\rangle$ is plotted with respect to $\tau$. The altitude is fixed at $y_{0} / \delta=0.18$. 


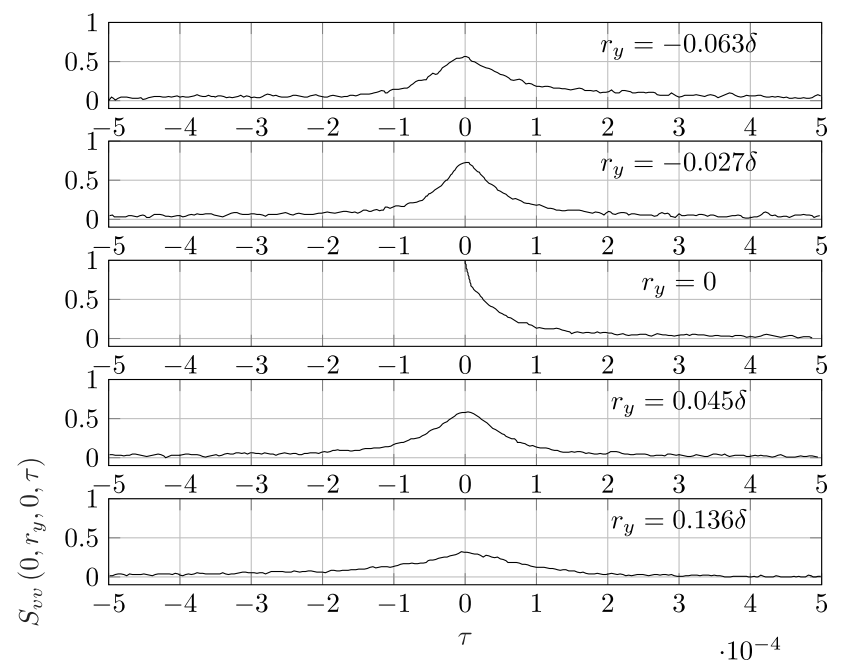

Fig. 10: Normal distribution of the velocity correlation. For each value of the distance vector $\mathbf{r}=\left(0, r_{y}, 0\right)$, the normalized velocity correlation $S_{v v}\left(0, r_{y}, 0, \tau\right) /\left\langle v^{\prime 2}\right\rangle$ is plotted with respect to $\tau$. The altitude is fixed at $y_{0} / \delta=0.18$. 


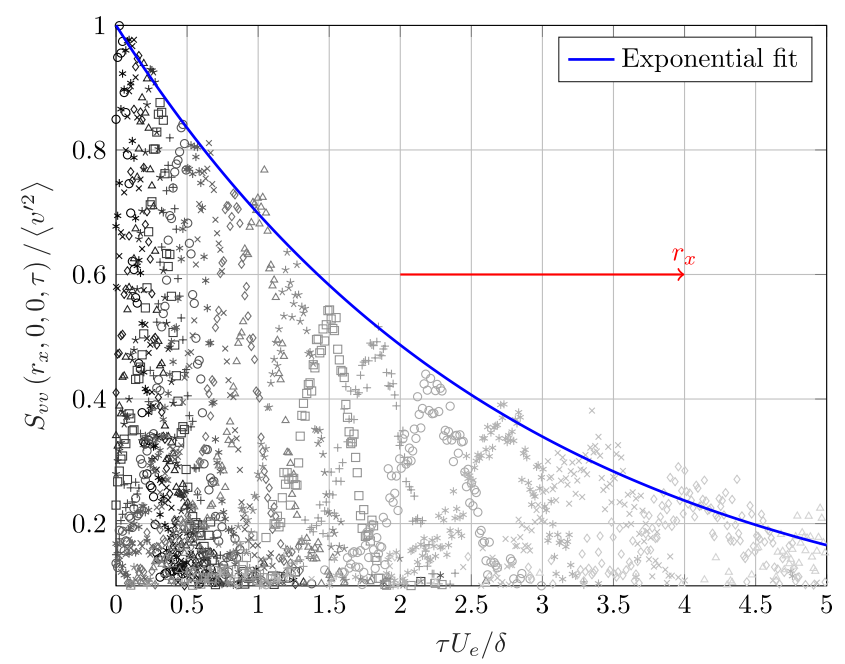

(a) wall-normal velocity correlation

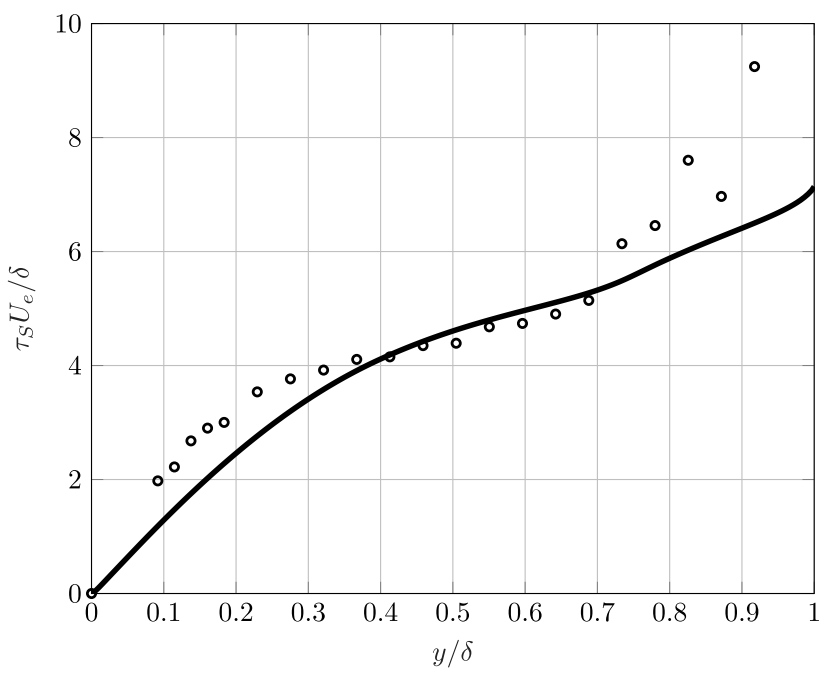

(b) time scale

Fig. 11: Decorrelation time scale. (a) evolution of $S_{v} v\left(r_{x}, 0,0, \tau\right)$ with respect to $\tau$. Black to gray colors indicate increasing $r_{x}$ values. Velocity correlations are obtained for $y_{0} / \delta=0.18, \mathcal{R}_{\theta}=7260$. (b) Profile of the decorrelation time scale $\tau_{S}$ in the boundary layer for $\mathcal{R}_{\theta}=7260$. 


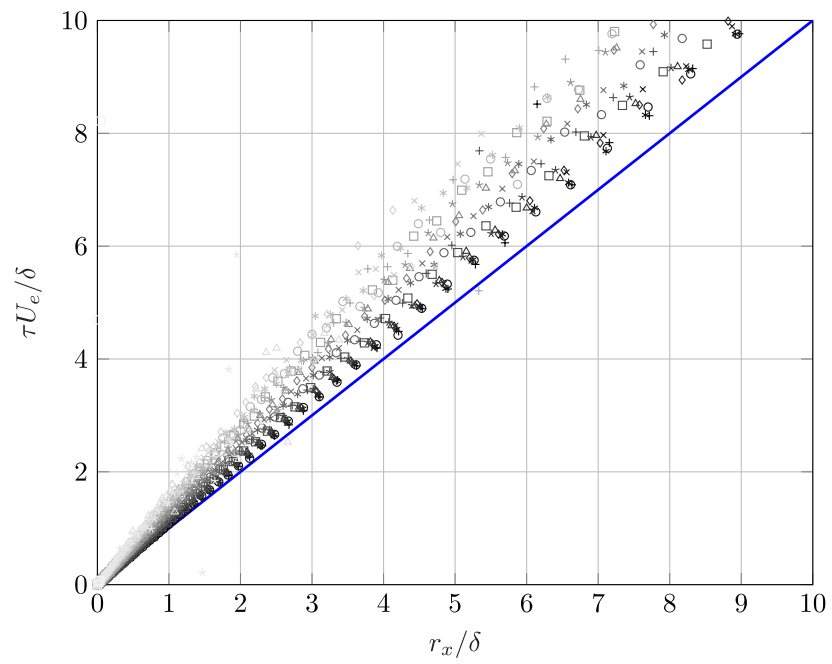

(a) $\max \left(S_{v v}\left(r_{x}, 0,0, \tau\right)\right)$

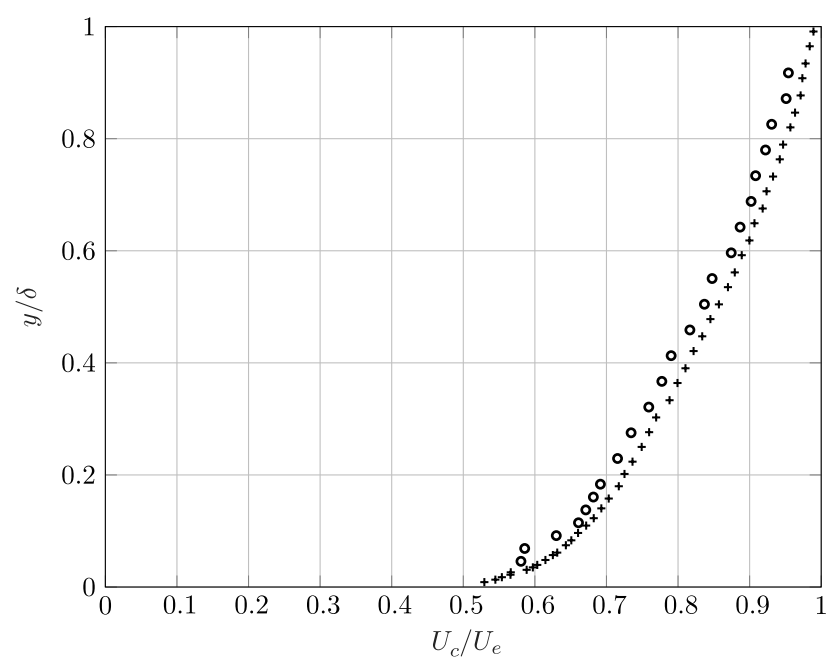

(b) convection velocity

Fig. 12: Convection of the wall-normal velocity correlations. (a) evolution of the peak values of $S_{v v}\left(r_{x}, 0,0, \tau\right)$ in the plane $\left(r_{x}, \tau\right)$. Gray to black colors indicate increasing $y_{0}$ values. The blue line represents the linear convection at constant speed $U_{e}$. (b) Profile of the convection velocity $U_{c}$ (o symbols) compared to the mean longitudinal velocity profile (+ symbols). 


\section{TABLES}

Table 1: Characteristics of the velocimeters

\begin{tabular}{|c|c|c|c|c|}
\hline & Wave-length & Beam power (each) & MV diameter & MV length \\
\hline \hline Blue & $488 \mathrm{~nm}$ & $20 \mathrm{~mW}$ & $180 \mu \mathrm{m}$ & $4 \mathrm{~mm}$ \\
\hline Yellow & $561 \mathrm{~nm}$ & $50 \mathrm{~mW}$ & $130 \mu \mathrm{m}$ & $3 \mathrm{~mm}$ \\
\hline
\end{tabular}


Table 2: Characteristics of the measured boundary layers

\begin{tabular}{|c|c|c|c|c|c|c|c|c|}
\hline$U_{e} \mathrm{~m} / \mathrm{s}$ & 25 & 30 & 35 & 40 & 45 & 50 & 55 & 60 \\
\hline \hline $\mathcal{R}_{\theta}$ & 2860 & 3780 & 4790 & 5860 & 6860 & 7260 & 8990 & 10570 \\
\hline$\theta \mathrm{mm}$ & 1.81 & 1.99 & 2.15 & 2.31 & 2.42 & 2.42 & 2.59 & 2.81 \\
\hline$\delta \mathrm{mm}$ & 17.1 & 16.6 & 18.0 & 19.0 & 19.8 & 20.1 & 22.0 & 22.8 \\
\hline$u_{\tau} \mathrm{m} / \mathrm{s}$ & 1.05 & 1.19 & 1.35 & 1.50 & 1.67 & 1.81 & 2.00 & 2.13 \\
\hline
\end{tabular}


Table 3: Maxima values used for the vector distance $\mathbf{r}$ in each direction for one-dimensional or bi-dimensionnal explorations

\begin{tabular}{|c|c|c|c|c|c|c|}
\hline & \multicolumn{3}{|c|}{ 1D } & \multicolumn{3}{c|}{ 2D } \\
\hline Name & $X$ & $Y$ & $Z$ & $X Y$ & $X Z$ & $Y Z$ \\
\hline \hline$D_{x}$ & $13 \delta$ & 0 & 0 & $2 \delta$ & $2 \delta$ & 0 \\
\hline$D_{y}$ & 0 & $1.5 \delta$ & 0 & $1.6 \delta$ & 0 & $1.6 \delta$ \\
\hline$D_{z}$ & 0 & 0 & $1.5 \delta$ & 0 & $2 \delta$ & $2 \delta$ \\
\hline
\end{tabular}


Table 4: Number of spatial correlations acquired for each kind of measurements and the associated data volumes

\begin{tabular}{|c|c|}
\hline$X$ & 1735 \\
\hline$Y$ & 561 \\
\hline$Z$ & 424 \\
\hline$X Y$ & 1638 \\
\hline$X Z$ & 1344 \\
\hline$Y Z$ & 1960 \\
\hline Total & 7662 \\
\hline Measurements & $3.82 \times 10^{9}$ \\
\hline Raw Data & $47 \mathrm{Go}$ \\
\hline
\end{tabular}

